\title{
D. D.
}

\section{DISSERTATIO ACADEMICA}

\section{SISTENS}

\section{ITER Iv CHINAM,}

Conf. Exper. Fac. Med. UDS.

\section{Moderante}

$$
\text { Viro Generofissimo }
$$

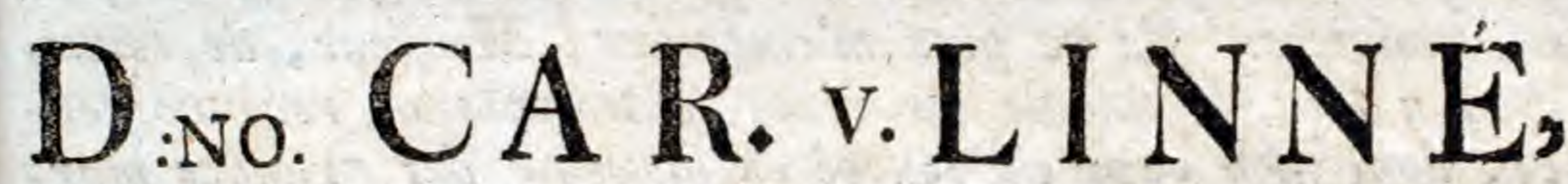

Archiatra Regio do Equiti Aur. de Stella Polnri Acad. Reg. Parif. Soc.

Publico Examini Submiffa

$A b$

ANDrEa Sparrman

Uplando.

In Auditorio Carol. Maj. d. xxx. Nov.

Anni MDCCLXVIII.

H. A. M. S.

ए58

UPSALIE,

Apud Jor. Edman, Reg. Acad. Typogr. 


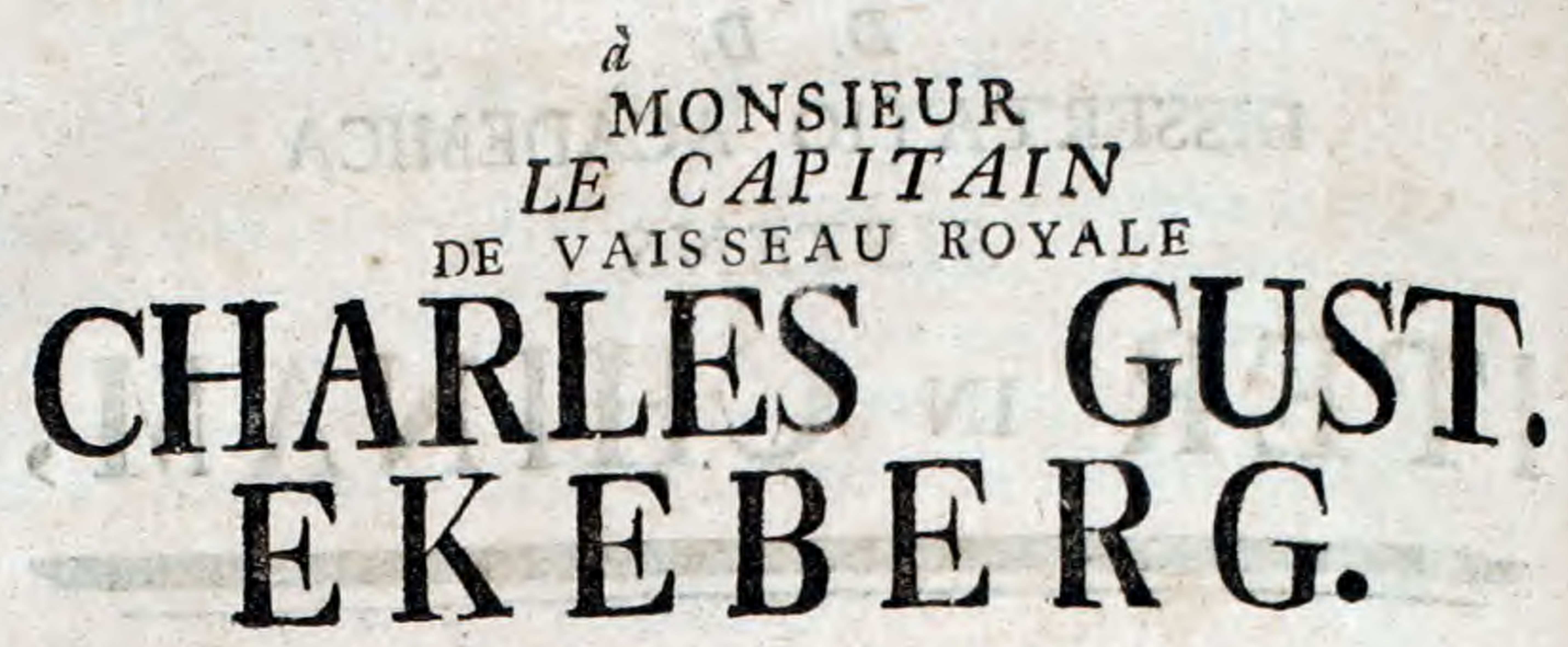

Te plaifir que vous aves pris d' enricbir l' biftoire naturel$\mathcal{L}$ le $\mathcal{F}^{2} d^{2}$ nutres foiences de decouvertes belles \& curi. eufes, donne moi de l' efpoire, que vous fcaures bon gré a liberté que je prens de vous offrir cette petite ouvrage, qui reprefente aux yéux du public les chofes curieufes, que j'ai vuës jendant mon voiage a Cbina, que je fis Sous vos aufpices. Fe ne puis miempecher, Monfieur, de temoi. gner a tout le monde les vives fentiments de reconnoiffence, dont mon ccur eft touché en fouvenir des grandes mar. ques de faveur, dont vous m'aves comblé pendant tout le temps que j'eus le bonbeur de vous fvivre par cette vafte efpace de notre Globe. F' aurois beaucoup de tort, $f$ je fe. rois affez temeraire de pretendre faire une digne eloge de us grandes merites: auffi ce feroit une cbofe fort fuperfluë: l' eftime que vous vous etes acquife de tout le monde fcavant, \& le credit que notre nation a cbez les peuples. d' Orient par votre belle \& Sage conduite, en font de te. moins inconteftables. Puiffies vous Mons: profiter long. temps de cette grande avantage, étre le luftre \& l' appui de votre patrie, le foulagement de votre famille, les delices de vos amis, enfin puiffe la fortune repondre a votre merite, et alors vous n' aures jamais fuiet de vous en plaindre. Fe fwis avec profond refpect

MONSIEUR

Votre trés bumble do trés obeifJant Jervitenls ANDRÉ SPARRMAN. 
Viris Admodum Reverendis \& Praclariffmis



Gevalia \& adjacentis Ecclefia Valboënfis PASTORI \& PRAPOSITO Meritifimo \& Infpectori Gymnafii vigilantifimo, Promotori Indulgentiffino.

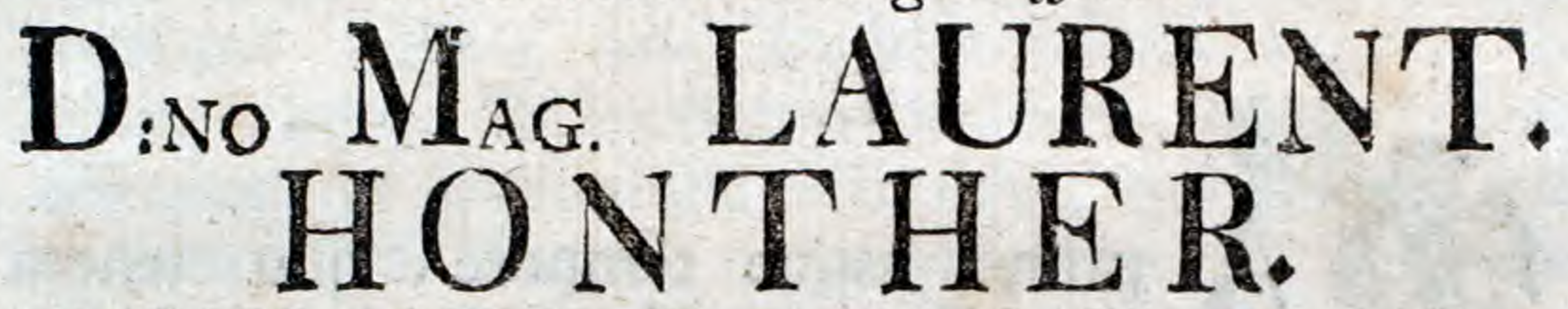

PASTORI ECCLES. Spongæ \& Jerfállæ Meritiffimo nec non PRAEPOS. - Contractus Sollentunæ adcuratifr. Promotori Propenfifjimo.

\section{Plurimum Reverendo \& Clariffimo}

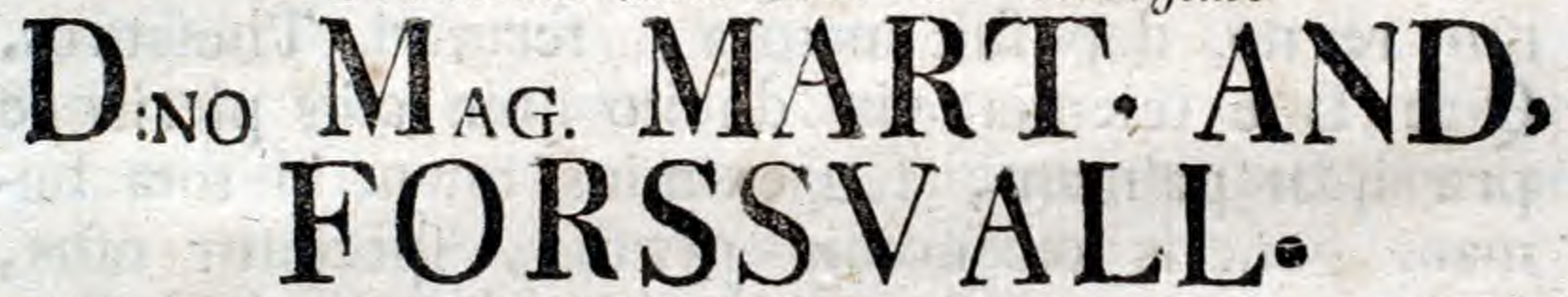

Animarum quæ in Tenftad Deo colliguntur PASTO: RI Dignifimo

Fautori \& Confangvineo Dilectiffimo.

Plurima \& eximia dum mentem fubeunt, quibus me ornaftis, favoris Specimina, me vobis debere video, quorum vel tautillam partem nulla fic fpes referendi. Opportune obortan occafionem, fummam qua flagrant penetralia, teftandi pietatem, latus arripui, Velit fummum Numen Vos in Annos bene multos fervare omnigena felicitate ornatiffmos, quod calidiffimis ab Eo efflagitare fufpiriis, fanctum mihi femper erit officium. Ad cineres usque permanfurus

NOMINUM VESTRUM

cultor humillimus

ANDREAS SPARRMAN. 


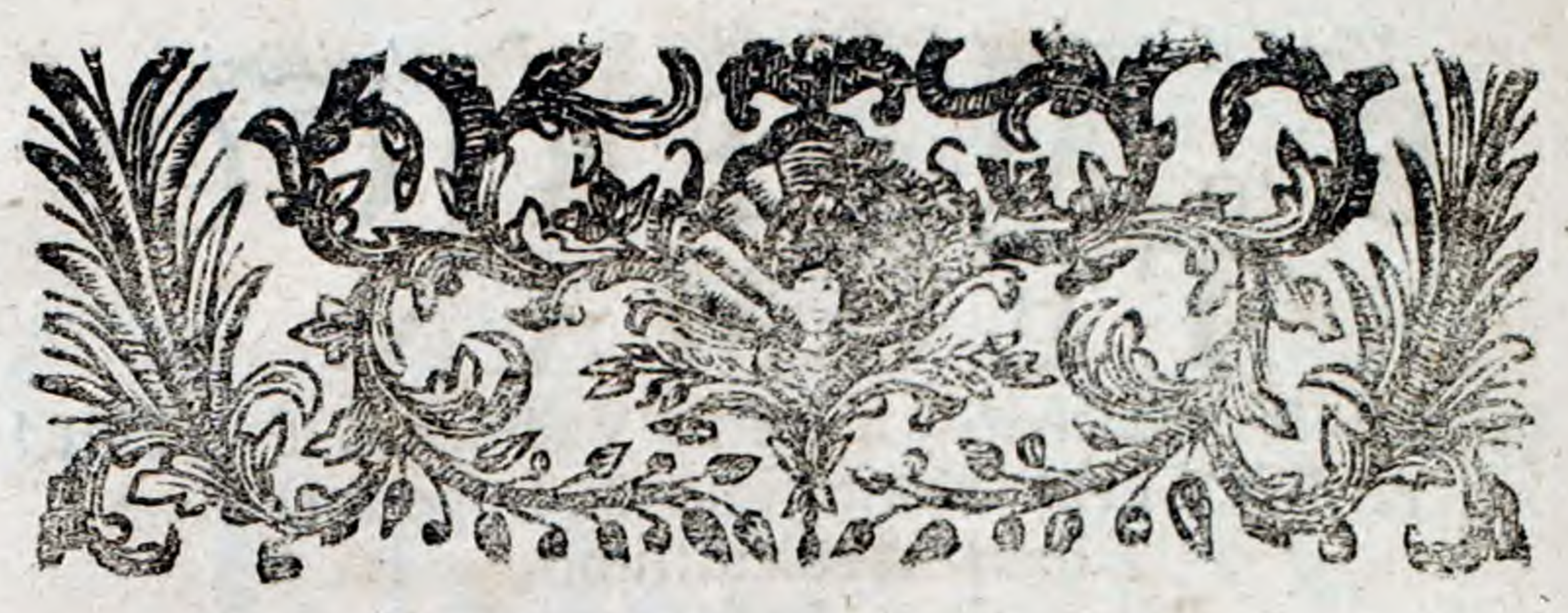

\section{Daves D. $D$.}

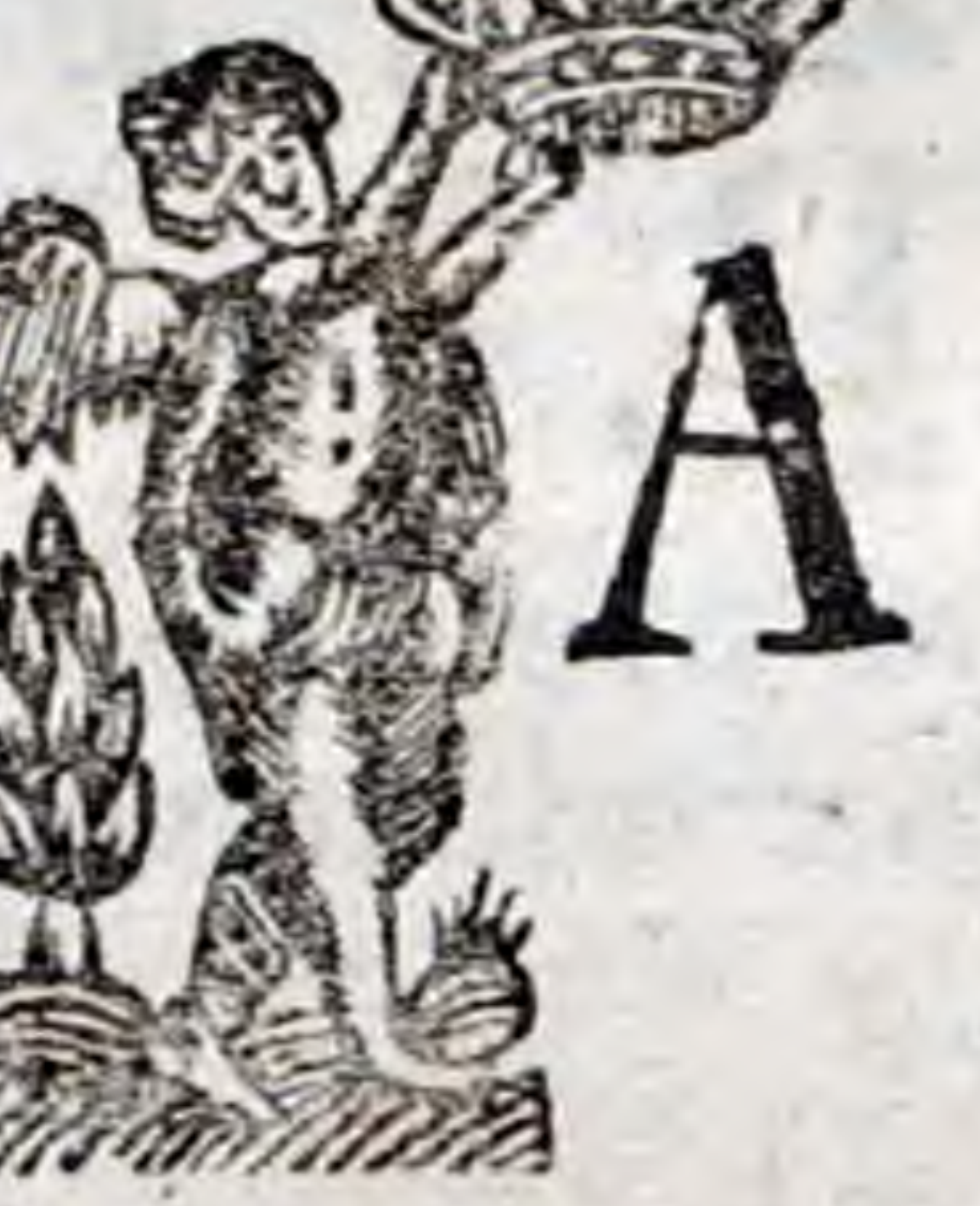

primo usque tempore, quo tenera mens incepit ad res in ftupendo naturæ opificio obvias attendere, incre. dibili flagravit fudio, pulcherrimos, guos in Vegetabilium atque Animalium regno depofuit natura, fcrutari Thefauros. Qux fane fcientia vix dubito quin aliis plerisque præripiat palmam, feu eximius quem in tota humani generis ceconomia præftat, fpectetur ufus, feu fumma, qua fuos cultores, in primis quibus in adyta ejus licet penetrare, perfundit voluptas. Felix mihi eft oborta occafio voti fieri compotem, dum Nobiliffimus Vir CAROL. GUST. EKERERG, in Claffe Reg. Navarcha, iter ad Chinam facturus, me fibi comitem fingulari favore voluit ad. jungere. Lxtus fane hanc accepi anfam, fummi Numinis potentiæ ac fapientiæ legendi veftigia, per viam quæ dimidiam, \& quod fuper eft, orbis terraquei ambit partem, alieni foli intuendi producta \& peregrinas luftrandi gentes: qux coram 


\section{解 ) 5(}

videndi nulla unquam fuiffet fpes, imprimis conFortio utens viri, qui absque dubio omnium Nautarum maxime eft fciendi cupidus, quique ipfemer prepria manu plorima rariora Animalia Pifesque delineavit*). Multum quoque ad hoc contulit fa. vor Patrum Academicorum, qui me Univerfalem hanc orbis Academiam vifurum, perbenigne fub. levarunt. Ante anni fpatium fospes relietas falutans mufas, \& Specimen Academicum in lucem edirurus, ut plerumque fieri folet, navita deventis narrans, fvavifimum mihi eft preteritos repetere labores \& qux in itinere vidi notatu digna, breviter commemorare. Oleum operamque me non exiftimo perdidiffe, fi vel unicam Plantam aut In. fectum detexero antea ignotum. Scientix enim cumuli formicarum inftar accreficune, qualibet formica paleam afferente. Itineris in Chinam exponere diffufam narrationem haud opere pretium du. $x i$, cum in his mihi otium fecerunt perplurimi, quos \& opporrunior occaffo \& felicior Minerva huic fpart $x$ reddidere aptiores. Sed paucis faltem narrabo, qux in quolibet loco inveni naturx producta, \&, fi nova quædam fuere, compendiofa defcriptione, adumbrabo, ut Doctores inde eliciant, gux ad artis perfectionem conferre intellexerint.

$$
\text { A } 3 \text { Die }
$$

*) Primus erat qui recte detexit TERMES fatale (S. N. p. 1015.) SCOLOPENDRAM phospboream (ib. p. 1064 .) omnium quoque primus fruticem THE $\mathbb{E}$ vivum in Europam transtulit (S. N. n:0 2. p. 365.) 


\section{\%) 6 ( :}

Die Dee XXVIII. Anni MDCCLXV. GOTHO. BURGUM reliquimus $\mathrm{L}$ ris in multum ma. re comitantibus. Infulas FERREAS pluresque regiones pratervedi portui GADES

Febr. XV. Anni MDCCLXVI. appulimus, ubi ad oppid. PORT. ST. MARIÆ horros Hefperidum, Citris \& Aurantiis cultis virentes vidi.

Mart. XX. folvimus e Gade \& vela ventis dedimus.

Mars XXV. Salvagos atque altiffimum mon. tem Pico TENERIFFA confpeximus.

April. XV. EQUINOCTIALEM tranfivi. mus.

Maji III. PELICANI Aquili quidam quafi fub nubibus harebant.

Maji VI. PḦ̈tTON athereus, Avis tropica alba loris tamquam duobus curfu per æthera fu. blimis ferebatur.

Maji. XVII. PROCELLARIE capenfes (Co. lumba capen/is) in confpectum veniebant, ab his ad Javam fere deducti,

Maji XXXI. brevia CAPENSIA bolide ex. plorabamus, ibique plures PROCELLARIAS pela. gicas vivas cepimus, nec non SQUALOS, quibus fe affixere ECHINEIDES, circum quos GASTEROSTEI Ductores natabant: Aves Jean von gents, qux inter indicia pracipua brevium capenfun numerantur, heic etiam, prater cæeteras aves minus notatu dignas, obfervavimus. 


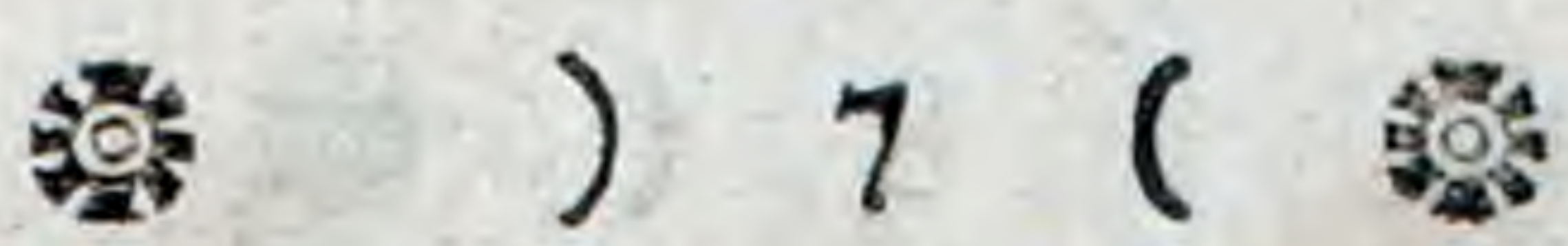

Junii. XVI. FUCUS natans fluctibus agebatur. Julii $\mathrm{X}, \mathrm{PH}$ ËTHON atbereus iterum volantare vifus eft.

Julii XIV. Infula JAVE fe nobis præbuit, per tres menfes terræ confpectu privatis. Julii XVI. Anchoram ad Infulam Neuv BAIJ de mifimus, ubi naturalia varia a me collecta funt, utpote: Amomum Curcuma ro. Sphex tropica **

\section{tunda}

Solanum indicum.

Panicum divaricatum

Sophora tomentofa

Rofa - - .

Dolichos trilobus

Elephanthopus fcaber

Mufa paradifiaca

Acroftichum furcatum

Gobius pectiniroftris *
Papilio Afcanius ***.

Phalæna Trax

- Petulca ${ }^{* * * *}$

- - Militaris

- - Fullonica, hæc ad genus Sphingis re. ferri debet \& inferi ante Alecto; fuperiores enim alæ funt anguftx \& poftice falcatæ.

- GOBIUS pectiniroftris: Pinna Dor $\sqrt{2}$ prior radiis V, extra membranam fetaceis; pofterior XXVI, Caude XVI. $A n i$ XXV. Ventrales radiis XII multifidis. Pe. ctorales XVIII, Dentes maxilla inferioris horizontaliter patentes, exceptis primoribus duobus longioribus erectis; Dentes maxillæ fuperioris plurimi, recti, fed VI primores longiores: intermediis magis diftantibus. Pifcis hic, qui nullis inftructus eft fquamis, in littore brevibus celeriter fe exilit faltibus.

* SPHEX tropica. Oculi non albi, fed nigri; Abdominis fegmentum fecundum bafi nigrum. Ale complicatæ Vefpæ. Maxille validæ, tridentatæ.

*** PAPILIO Afcanius (Eques) caudatus ater, 


\section{) $8 .($}

Ifis Hippuris

Nautilus Pompilius

Trochus niloticus

Chama Hippopus

OAtrea nodofa

pacella Nubecula

Nerica Hiftrio

Voluta turbinellus noftra caret columella plicata.

Oitrea formicata valuata $E \% c$.

Madrepora Fungites

- - Cancellata refert
Madreporam murica. tam, fed rami cancel. latim anoftomofantes \& decumbentes.

Turbo ftriatulus

Arca antiquata

Venus mercenaria

Strombus millepida

Murex turbinellus

- - tribulus

- ramofus

- Erinaceus

Conus ftriatus

Bulla Ovum.

lis fuperioribus albido lineatis; inferioribus fubtus ma. culis VIII coccineis.

Magnitudo mediocris, Caput fangvineum. Tborax lateribus fubtus fanguineis. Abdomen atrum, fubtus fangvineum \& nigro variegatum. Alce primores atra, concolores, fecundum venas albido lineatr \& quidem fubtus magis, quam fuora. Alce poficice atra, repandx, obtufe, fubcaudate, maculis marginalibus fubrotundis VIII late coccineis. Pedes nigri.

**** PHALANA Petulca fpirilingvis levis, alis fufcis: fuperioribis fafcia, inferioribus difco albis.

Statura Phal. Facabee. Caput flavum: fronte, vertice, \& oculis nigris. Tborax operculis duobus, lanceolatis, margine flavis. Ale fuperiores concolores, fufcx: Fafcia in medio alba, potice repanda; ad bafin
ala ftria aliquot alba limbo fulco. Ala inferiores fil- 


\section{: 9 (}

Jul. XXII. dum infulam, quæ PRINTS EYLAND appellatur, præterivimus, Javani ad nos ad. duxerunt Simias, Pfittacos, Gallinas \& Viverram jchnevmonem.

Jul. XXX. Fumus cx fodinis ftanneis ad MO. NAPIVI confpiciebatur.

Augufti X. infulam PULOCANDOR præterveEti . Aug. XXIV, ad MACAO devenimus, ubi introitus eft ad urbem Canton.

Aug. XXVI. Anchoram in CHINA haud procul ab urbe Canton jecimus. Sequentia heic mihi obvia fuere naturalia.

ORYZA fativa, \& ARUNDO faccbarifera mixtæ, fluxui \& refluxui aquarum expofitæ, degebant. Plante antem ferquentes in aridis locis collectx.

Thea Bobea

Ixora coccinea

Rhamnus lineatus,

Becchra frutefcens

Triumfetta Bartbramia ,

Urena procizmbens

Hedyotys fruticofa

Barleria crijzata,

Fructus etiam fequentes

Bromelia Ananas

Curcurbita Citrullus

Tamarindus indica
Sinapis Bralficata,

Polygonum cbinense, .

Torenia afiatica,

Aralia cbinenfis, fed tota glabra,

Verbefina calendulacea, Hedyfarumbiarticulatumn
Citrus decumanus

-. Aurantium

B

LEMUR

fcæ difco amplo, albo, ad marginem interiorem excur; rente. 


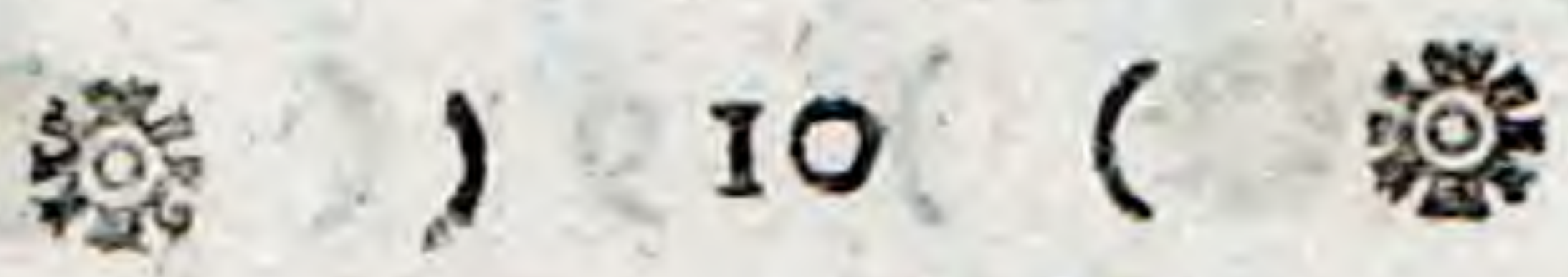

LEMUR Mongos. Corpus totum gifeum. $F_{a}$ 。 cies nigra: Mare gula ferruginea; Femina gula \& ano albis.

TRICHIURUS LepturtLs, CYPRINUS aura. tus, CLUPEA chinen/is, PHASIANUS Gallus tam - - Lanatus quarn - pufillus.

Papilio Hecabe

- Paris

- Leucotboë

- Pannmon

- Demoleus

- Argyrius*

- Pyrene

- Alnzana

- Orithya
- Lemonias

- Midamus

Phalæna Atlas

nigrella**

Altica ***

Blatta orientalis

Fulgora Candelaria

Spinx Porcellus captus eft

Pha.

* PAPILIO Argyrius (nymphalis) alis dentatis fulvis, nigro maculatis, fubtus ocellis quinque liturisque argen. teis. Facies Pap. Nipha. Ale Primores concolores, lutex: pundis nigris plurimis, præter lineolas transverfas nigras ad marginem exteriorem. Alce poftice fuprà pri. moribus fere fimiles; fubtus vero magis pallidx, variegatæ maculis grifeis præter ocellos V, cum lituris variis argenteis.

** PHAL, ENA Nigrella (Tinea) alis atris, ftrigis albis : anteriori fimplici; pofteriori circumflexa (locan. da polt, 335.)

Corpus magnitudine Phal. Evonymella, atrum. Antenne fetacea, nigra. Abdomen cingulis linearibus, auguftiflinis, VII, albis. Ale concolores: fuperiores deflexæ, atra: ftrigis duabus, anguftiflimis, albis, ante 


\section{\% ) II ( sis}

Phalæna bicincta ${ }^{* * *}$ in ipfa navi dum in macrops *****, China commorabar; u$\mathrm{B} 2$

trum

riore verfus bafin transverfa, qua vix tangit marginem inferiorem. Pofteriore paulo latiore, a margine interiore ad inferiorem circumflexa, cum punito albido ad flexuram anteriorem. Ale inferiores nigres: ftriga alba in medio. Collare \& Frons alba. Pedes fpinofifimi, fubtus albidi.

*** PHALENA Altica (No Zua) fpirilingvis lavis flava, alis fuperioribus maculis duabus coeruleis margine caruleo connexis.

Corpus magnitudine \& ftatura Phal. Jacober, flavum Oculi nigri. Antenne fetacex, fufcre. Tibie \& Plan. te nigro-carulefcentes. Ale fuperiores margine longitudinali coeruleo; macula magna carulea in medio a. lre, margini cerruleo annexa; macila altera cxrulea, verfus apicem, lunata, margine interiori itidem annexa; Hinc area prima alæ flava; altera area flava, pone medium; tertia macula flava in apice. Thorax humeris caruleus. .

**** PHAL FNA bicincta) (Notua) fpirilingvis fublrvis, alis grifeis nebulofis; inferioribus fupra fafciis duabus albis;

Magnitudo Phalænæ Pacte. Corpus grifeo-æneum, Ale deflexæ: fuperiores fupra antice \& poftice grifex, plaga intermedia cinerea, qux antice \& poftice diftinCta linea nigra undulata; fubtus cinerex, fafcia trans. verfa, alba. Alre inferiores fupra fufcr: fatcia in medio alba \& margine poftico albo; fubtus cinerez: angulo ani nigro, fed terminato macula alba,

*****PHALÆNA macrops (Attacus) feticornis fpiriligvis, alis fufcis atro undulatis: fuperioribus fupra ocelto ferrugineo; fultus albo maculatis. 


\section{\% ) $12(\%$}

trum vero Chinetılis fuit aut ex Europa cum na. ve allata, non facile dixero.

Anno MDC̈CLXVII Jan. XXI.CHİNAM reliquimus. Sterna folida ad Javam usque nos eft comitata. Febr. IX ad Infulam NORD EYLAND an. chora tenebamur, ubi fequencia nccurrebant. Lobelia Plumieri. Palpalum diffectum. Clerodendr. fortunatum Apluda mutica. Convolvulus Pes capra, Urena lobata. in littore. Vitex trifoliata,

Papilio fava * P.. Canidia ${ }^{* *}$ Cimex Stockerus

Onifcus Afilus *** Scarabæus oricbalcus****

Sphinx

Corpus fecundde magnitudinis, fufcum. Antenne fe. tacex. Palpi ereiti, comprefli, apice filiformes. Ale omnes dentatæ, fupra fufca, atro undulata: fuperiores ocello, in medio, magno, ferrugineo, iride nigro pupillaque lunari atro-cærulea Subtus fufcæ maculis aliquot albis in medio. Inferiores magis fufcr, maculis nigris fagittatis; fubtus punctis fubfagittatis albis adfperfx. Similis Phalæna crepufculari, Clerk tab. 53 fig. III. $I V$. fed magis fufca.

* PAPILIO Fava (Dan. cand.) alis albis poftice nigris, fubtus bafi fulvo maculatis poftice albido punctatis.

Corpus ftatura Pap. Braffica: Alee omnes Jupra dimidio antico alba, poftico nigra: Primores verfus apicem lituris tribus albis. Subtus nigra: Prinzores bali fulvæ, apice lituris tribus albis. Poftica fubtus bafi ma* culis VI vel VII fulvis, convergentibus, margine po- 


\section{(5) ) I3}

Sphinx Polymita *

Cancer minutus

Buccinum rufum

Trochus Perdix

Tubipora mufica

Trochus perfoectivus

Gorgonia Abies

- - anceps

- ventalina

$\mathrm{B}_{3}$

Febr.

ftico punCtis VI. majusculis albo-fulvis feu omnibus alis interrupte albis.

** PAPILIO Canidia (danaus cand) alis integerrimis rotundatis albis: primoribus maculis duabus nigris; pofticis fubtus bafi flavis.

Statura \& magnitudo Papilionis Rapæ. AL 压 primo. res concolores, alba maculis duabus nigris, fed fupra apice nigre. Ale poffice fupra albx maculis marginalibus VI, nigris, quarum anterior reliquis multo major: fubtus pallide cinerafcentes, margine antico flaviffimo. *** ONISCUS Afilus hujus fegmenta habent us trinque ad latus duas fpinas; alteram juxta bafin exifam, alteram juxta apicem lateris protrufam.

**** SCARABÆUS oricbalcus fcutellatus muticus aneus glaber, fubtus ferrugineo varius:

Corpus magnitudine \& ftatura Sc. Eremitx, fed fterno parum porrecto, Caput quadratum marginibus lateralibus elevatis, fed angulis anticis mucronatis. Tho rax lævis, pontice trilobus: lobo medio productiore, fupraque impreffo, (fcutellati reliqui non funt Thorace poftice produatiore). Scutellum minutum. Elytra levia. Sts= btus niger abdominis lateribus ferrugineis. Femora ferruginea, apice nigra, Color totius fupra viridi zneus nitens* SPHINX Polymita (Afcita) abdomine luteo fafciis fex. nigris, alis fuperioribus maculis feneftratis quinque, inferioribus duabus

Magnitudo Tipula majoris. Corpus luteum, macus: 


\section{被 ) 14 ( 总}

Febr. XV. Navem folvimus, \& à Nord Eyland dilceffimus,

April. XXII. CAPUT BONE SPEI in confpectum venit, videbam heic quidem natantem UL. VAM bucciniformem (Trompetgrås), eandem autem cupidus licet colligere, fiquidem adhuc non rite fuit determinata, vetuit navis curfus.

April. XXIV confpectui fefe offerebat Colum. na aquatica magnitudinis infolitæ \& multo major alia, quam in agro Oryzæ chinenfi prius obfervavi, quaque explofo tormento evanuit.

Maij. XI. Anchora jactata eft ad infulam St. HELENE, ubi PORTULACE oleracea ingens copia fponte crefcebat.

Maj, XVII. capti funt SCOMBER Pelamis (Bonet), SCOMBER Thyznus (Albacora), CORY. PHENA Hippurus (Dolphin); Hi omnes agitabant Exoccetos volitantes, ut in navem decidui fuerint capti. DELPHINI Delpbides, SEPIE Loligines \& LABRI ariftafti.*

lis \& fafciis nigris. Ale fuperiores nigræ, maculis hya. linis: primo una, deinde binæ \& demum binæ; Ale inferiores minuce, nigræ macula feneftrata gemina.

* LABRUS ariftatus (Dubii generis pifcis)

Pinna Ani radiis XXV: primo brevifimo. Pinna Dor $/ 2$ XXXII. Pectorales XII. Ventrales VI, Color argente. us. Corpus ovatum, valde compreffum, transverfim ftriatum: fquamis brevifimis, fed latiflimis, latere utroque ariftatis. Opercula lrvia. Facies Oftracionis, fed den. tes fimplices \& fparfi: primoribus inferioribus duobus longioribus. 


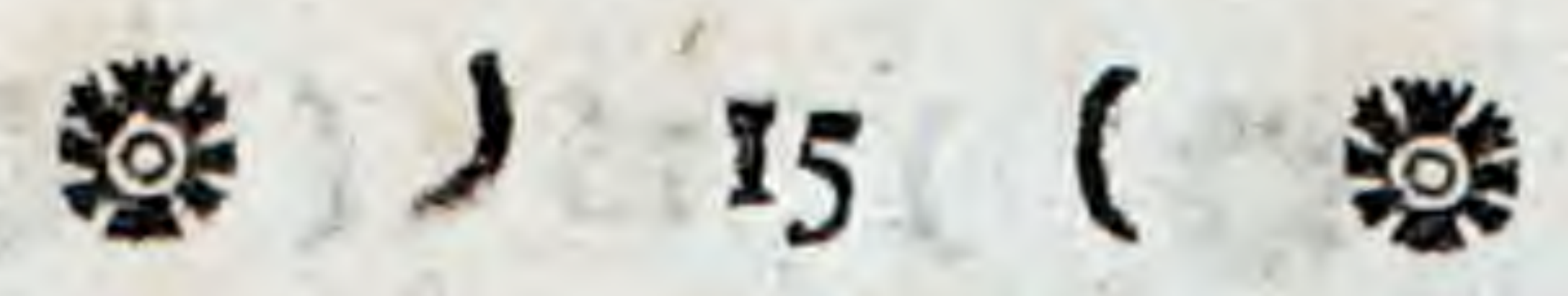

Mai. XXV ad infulam ADSCENTIONIS per. venimas, ubi in litrore numero 79 TESTUDINES Midas cepimus, \& PORTULACAM olera. cean collegimus. Heic PELICANUS occidentalis confpiciebarur. BALISTES Vetula hamo capta fuit; hac coloribus formofa, fed efui infvavis.

Maj. XXVI hine poftguam nave in altum mare devecti, maximam FUCl natantıs (Sargazo) copiam undis agi vidimus. In hoc SCYLLAAM pelagicam \& CANCRUM Neptuni ** coepi. HOLOTHURIA Pbyfalis quoque capta, cujus Cirri urtica vehementius urunt.

Jul. IV. MEDUSA Velella nobifcum vela dabat ventis.

Jul. XIX. TESTUDINEM Midam in aqua dornire vidi.

Julii. XXI. Anchora ad FAYAL, una Infula. rum Azoricarum jacta, inde Patriam petimus tandemque Augulti XVI attigimus, ubi paucellas has obfervationes in lucem edere Iubet; quominus

au*

** CANCER Neptumi (macrourus) thorace antice pofticeque trifpinofo. (poft Homarum 74. locandus)

Corpus magnitudine Tipula. Tefa figura fructus Burfæ paftoris, oblonga; antice carinata \& anguftata fpinis tribus porreftis, quarum media longior, fupra caput extenfa; Poftice tefta emarginata margine deflexo armato fpinis tribus, quarum laterales rectr, media vero brevior erectior. Corpus pone teftam lineare, longitudine ipfius teftr, terminatum Cauda latiore, obovata, ferrata mucronibus VI. parvis. Oculi pedunculati. 


\section{部) $16($ (}

autem plures tibi B. $\mathbf{L}$. novas queam offerre fpecies, varia me impediunt. Haud enim facile fuic fub ardente fole longos conficere curfus; nec Chinæ campos excurrendi libertas advenis concedi. tur; nec ñ JAVA ferocifima Animalia ut Croco. dyli, Tigrides \&c. ac Frutices fpinis aculeisque ar. maræ, quibus littora funt confertim obfita, fecurum commodumque curfum permittunc.

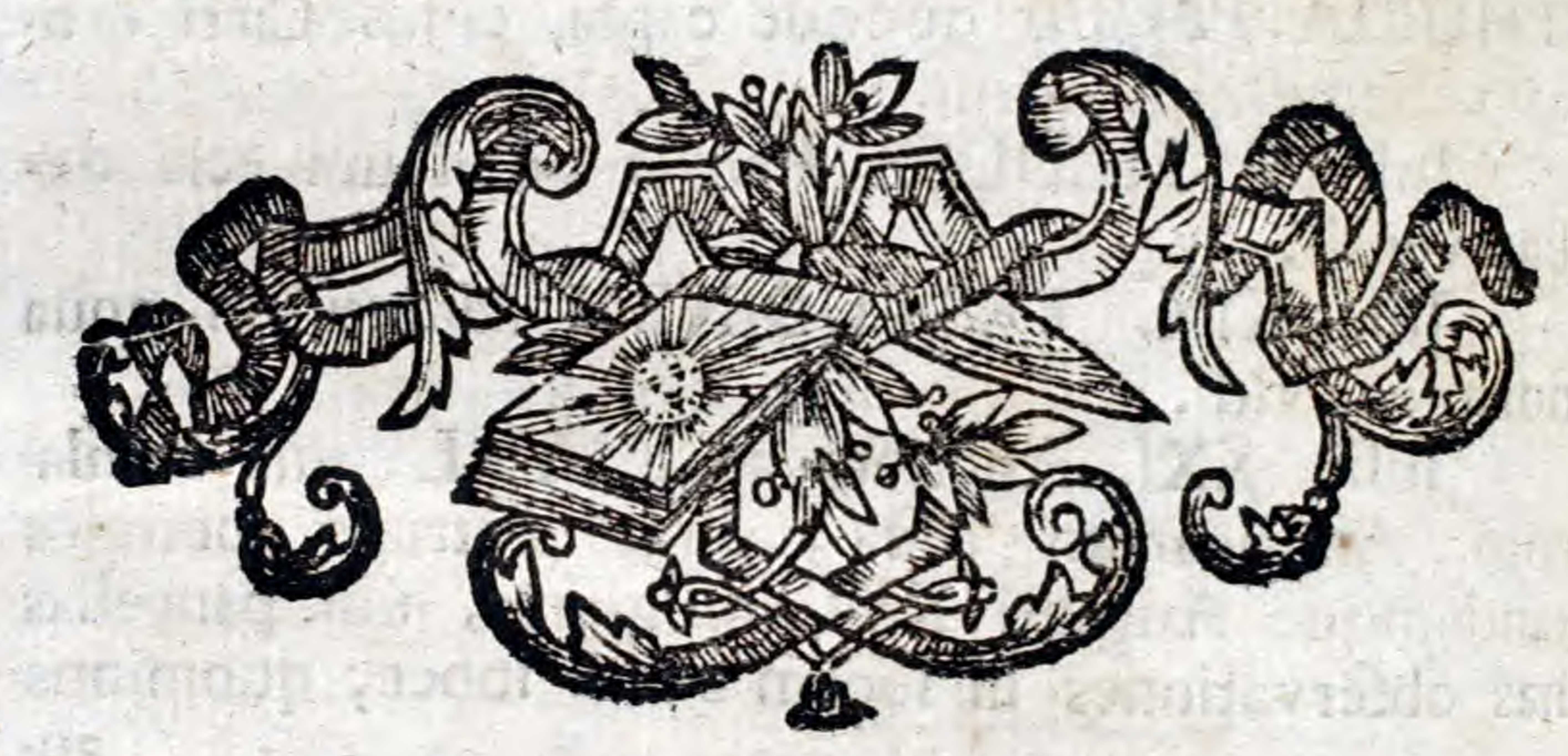

